\title{
Role of Social Workers in the Rehabilitation of Addiction at Correctional Facilities Centers in Karachi, Pakistan
}

\author{
Dr. Sakina Riaz, \\ Corresponding author, Assistant Professor, Department of Social Work, University of Karachi. \\ Maliha Sahar, PhD Scholar, \\ Department of Social Work, University of Karachi.
}

\begin{abstract}
Substance abuse have become common in recent years which is why there are a number of cases being reported in the Correctional centers of Karachi regarding their special treatment and effective care. Since there are different interventions in reducing the number of addicts, social workers have been continuously regarded as prime support to such victims because of their skills and expertise in treating and improving their health concerns. The main purpose of the research is to highlight significant roles of social worker in controlling drug abuse so that society can face a positive transformation. It is explored that majority people in Karachi, Pakistan are subjected to use drug on daily basis because of immense societal pressure and influence of other communities. For investigating core initiatives that social workers take to improve such cases, mixed research strategy is used. Primary data is collected through survey in which 200 participants were approached from three different Correctional Centers in order to provide quality knowledge regarding the subject. Moreover, secondary data was collected through reviewing different online sources. The findings of the research suggest that the role of social workers is to provide support, quality care, consultation, and training to minimize drug effects. The conclusion drawn from the research also illustrates that almost all of the rehabilitation centers have employed social workers who have prior experience in handling drug addicts and they are credible enough to work in Correctional Centers.
\end{abstract}

Keywords: Drug Addicts, Social Worker, Social Work, Substance Use, Pakistan, Awareness, Training

DOI: $10.7176 / \mathrm{JESD} / 10-11-10$

Publication date:June $30^{\text {th }} 2019$

\section{Introduction:}

Around the world, Addiction and its continuous use have been widely increased, which is now known as perennial problem in the society. Among these drugs, Narcotics is one of the substances that is used in medical practices while it is not legally available for personal use without the permission or prescription of a physician. Such types of drugs are commonly taken by individuals for a number of reasons and thus, when its rate of consumption increases, a person is likely to become addicted (Daley, Dennis and Marvin, 2013). Basically, the term "addiction" is known to have unmanageable background that has harmful effects when it is not controlled in right time. People who have certain patterns of taking drugs in their routine are known as drug abusers and therefore, they are also recognized as drug addicts due to continuous consumption of substances that have negative side effects on health. The main purpose of developing following research is to understand how social workers are contributing in the society to control growing trend of addiction. A number of studies have been explored in this regard to investigate key practices that social workers perform in their professional services so that a healthy and prosperous society could be created. In addition, Pakistan comprises of diverse culture where a number of different communities are resided, which practice their rituals and cultural activities according to their norms and values. For this purpose, young generation specifically is being influenced by such changes with which positive and negative characteristics are prevalent in the society (Brown, 2015). With such changes and social impacts, many young people and adults are found involved in adopting practices which are considered illegal and immoral in Pakistani society. 


\subsection{Addiction in Pakistan}

Pakistan is one of the prominent nations in the world where the society is mainly furnished with numerous resources through large imports. In Pakistan, both males and females are reported to involve in such activities that are considered illegal and unethical but are practiced to overcome their social and economic challenges (Gao, Huan and Jianhong Liu, 2016). The main reason behind such trends among the society is transitional change which is associated with challenges that have influenced their mindsets and behavior to the greatest extent. With respect to Pakistani citizens, they often receive socio-cultural and economic threats, struggle from educational particulars, ineffective coordination, societal pressures and family responsibilities, religious restrictions, and community norms and values. Munir et al. (2019) explains that such negativity and immoral activities in the society have lead Pakistanis to adopt practices that are basically related to social evils.

It is also studied in another article developed by Jafari et al. (2015) that drug addiction has prevailed among Pakistanis for many years which is referred as social stigma due to going against Pakistani social values and primary position that religious person holds in the society.

The important part that is being investigated in most researches is to control prevailing drug activities among youngsters so that a drug-free society could be maintained. In this way, the primary steps are to understand its root causes and intervention of rehabilitation activities that are subjected to control drug abuse at large. Khursheed and Chandra Madhudas (2017) address that in order to eliminate growing trends in the society, it is highly important to assess the culture first and then investigate relevant protocols that would help in achieving drug-control objectives in a directed way. The author also highlights that a person who is the head of a family is a primary role of the society which is termed as home architect. Therefore, if he/she is addicted to substance abuse then whole family is affected which is why a number of socio-cultural and economic problems are growing each day.

By studying the patterns of drug use, it is found that Pakistanis under psychological and socioeconomic distress including any mental health issue, poverty, and lack of hold in the homemaking decisions are some of the prevailing factors that indulge them in such practices (Scheff, 2017). With such activities, they undergo depression and frustration that compels them to take drugs and other substances which are easily available that can be purchased without any prescription or permission. It is also studied that these abusers feels pleasure and satisfaction in consuming such substances that allow them to escape from life's challenges without being aware of resulting consequences. Thus, both males and females are found to be involved increasing drug addiction patterns that have affected Pakistani society to the greatest extent.

\subsubsection{Causes of Addiction in Pakistan}

Since Pakistanis have been investigated in terms of assessing their behavior and attitude towards seeking drugs and related substances, the results declare that there are number of reasons that cause Pakistanis to undertake excessive drugs in order to influence their lives and lessen psychological impacts at large. However, lack of awareness and attention from family and community also increases prevalence of substance abuse that largely affects the character and health in a broader context. Soomro et al. (2018) have highlighted some of the primary reasons and root causes of substance abuse that provoke them to consume excessive drugs and substances that are dangerous for their health and survival. Some of the core causes are enlisted as follows:

\subsubsection{Pleasure}

In Pakistan, abusers tend to abuse themselves from drugs because of seeking instant pleasure and joy. According to McGovern and Stephanie Sarabia (2018), drugs have special ingredients that have capability to trigger emotions and psychological characteristics due to which the victim feels pleasure and joy. However, as different types of drugs are available and individuals consume varying substances, pleasure and sensation is also felt at different rate. In order to explain the cause, Crossin et al. (2019) discusses that cocaine is one of the types of drugs which influences self-confidence, higher energy level, and internal power that enable individuals to perform activities in a most active way. Similarly, heroin is another type that brings pleasure in the life while has also strong characteristics. Marijuana is also a drug which is when taken increases satisfaction, happiness, and relaxation.

\subsubsection{Relief}

According to Bandiera et al. (2015), Pakistanis have to suffer from immense societal pressure due to which they seek for satisfaction and relief in other harmful and dangerous practices. It is identified that most of the patients are diagnosed from major psychological disorders which affects their growth and development at large. Such problems are identified as social anxiety, stresses, mental disorders, depression, frustration, and even insomnia as well. In order to overcome these problems, abusers tend to consume drugs excessively that lessens their loneliness while improves mental stability at large (Daley, Dennis and Marvin, 2013). The patients use drugs in a consistent pattern to seek relief so that they can perform their activities and responsibilities without any inconvenience. Basically, stress is one of the psychological patterns that are felt with continuous pressure while it is lessened when individuals take drugs in large quantity.

\subsubsection{Enhancing Abilities}

Societal pressures and demand of unique abilities are also subjected to influence mindset of individuals due to which they have to take such elements that are not only dangerous for health but also affects environment as well. 
In this way, Swensen (2015) stresses that with an increased societal pressure and competition among individuals, people are compelled to take drugs so that they could perform and possess abilities that the society wishes to see in them.

\subsubsection{Role of Social Workers at Correctional Facilities Centers in Karachi}

Among the practices to treat and cure patients, social work is also a most prominent and influential profession that is aimed at providing beneficial support and care to the people who are in need of helping hand. Abramovitz (2017) defines "social work" as a professional activity in which professionals provide attention to individuals who are under immense stress or involved in activities that are not acceptable in the society. Basically, social work is linked with transferring services to the society to achieve positive outcomes in terms of improved circumstances and conditions, as well as positive attitudes that are important for maintaining standards of the society (Daley, Dennis and Marvin, 2013). The core of social work practice contains set of principles and values which ensures that all the social workers have to perform their responsibilities for individuals while also provide care to those who are dependent on them.

There are not enough rehabilitation centers for substance abusers who are residing in the prisons of Karachi. Recently Sindh government came up with a plan of turning prisons in correctional facility centers that would totally focus on the rehabilitation of those individuals who are in desperate need of rehabilitation and want to leave their addiction of substance abuse. By introducing these types of reformed programs the abusers would get a chance to live a normal life. These programs are providing a healthy environment to the prisoners as they are encouraged by the social workers to involve in a crime free life with responsibilities and dignity.

These programs are racism free, as they encourage the social workers to not discriminate any abuser on the basis of her/his race, crime, color, language, sect, ethnicity and family status. With the help of social workers other individual and groups are also involved such as; Sindh prisons minister, Sindh government secretaries for home, law, health, higher education, social welfare, women development, agriculture, and information technology individuals.

Social workers in the correctional facility centers in Karachi perform their roles and responsibilities according to nature of case and requirement of the individual. According to Singer and J. Bryan Page (2016), drug addiction treatment is carried by social workers in Karcahi with respect to providing support and care to the individuals in such a way that they again start living healthy and prosperous life. It is also referred in the study that main role of a social worker in such cases is to look for ways which are beneficial for substance users in order to lessen their addiction, as well as console their families so that individuals can overcome their problems as early as possible. The concerns related to substance use addiction involve certain psychological and physiological disorders which require more attention and care from practitioners so that they could recover from their addictive behaviors.

From research evidence Schnall (2016), it is analyzed that adult social workers i.e. specialized in managing elderly people, are found to handle cases that involves two to three people affected by their own substance use or any other person induced in them. With the growing demand and evidence regarding increased rate of addictive patients, social workers in Karachi are struggling hard to respond to such cases so that individuals could be given care and attention in an effective way. It is witnessed that some of the social workers have performed their responsibilities by giving them care and support so that they could involve themselves in other activities that are useful for their health (Lipset, 2017).

\subsection{Study Objectives}

The aim of this research is to explore primary intervention of social workers of Karachi in managing substance abuse so that individuals can live healthy and prosperous life. The primary objectives of the study are to:

1. Understand prevalence of substance use among Pakistani prisoners

2. Identify major causes of substance abuse that have provoked these individuals to consume excessive drugs

3. Define and investigate concept of social work in the light of providing support and care to individuals

4. Determine primary role of social workers in rehabilitation of addicts mainly in Karachi

5. Propose approaches to improve social work activities for improving state of addictive individuals

\subsection{Hypotheses}

1. Social workers in rehabilitation centers are source of health care and support to drug addicts residing in Karachi

2. Social workers have to train and develop awareness among community and families

\subsection{Methodology}

The study employed mixed research strategy in which both participants, as well as past research articles, were used to gather authentic and most reliable data. The purpose of using quantitative strategy is to assess and analyze quantitative data which helps in estimating right percentage of responses that are important for drawing appropriate conclusion. However, qualitative strategy is also important as it gives in-depth knowledge of the topic upon which the research is conducted. The researcher approached three Correctional Facility Centers in Karachi namely 
Central Prison Karachi, District Prison Malir, and Central Prison for Women. From these centers, the researcher selected a sample size of 200 participants who have provided valuable information that is important to conduct the study.

\subsection{Findings}

Table 1 illustrates frequency distribution of participants according to age groups. The table describes that highest number of participants belonged to 26-30 years age group, which is $50 \%$. The second highest numbers of participants lie under the category of 20-25 years age group, which is $27.5 \%$. However, only $12.5 \%$ of the participants were from 31-35 years age group while 7.5\% of the participants belonged to 36-40 years age group. The least number of participants were from 41 and above year's age group, which is $2.5 \%$.

Gender distribution of participants is illustrated in table 2 which indicates that highest percentage of the participants was male, which is $75 \%$ while only $25 \%$ of the females recorded their responses against the survey.

Table 4 Social workers provide valuable assistance to drug addicts to improve their health and life

\begin{tabular}{|c|c|c|}
\hline Response & Frequency & Percentage (\%) \\
\hline Strongly Agree & 123 & 61.5 \\
\hline Agree & 32 & 16 \\
\hline Neutral & 25 & 12.5 \\
\hline Disagree & 20 & 10 \\
\hline Strongly Disagree & 0 & 0 \\
\hline Total & $\mathbf{2 0 0}$ & $\mathbf{1 0 0}$ \\
\hline
\end{tabular}

Table 3 indicates responses of the participants regarding role of social workers towards assisting drug addicts. According to the results, $61.5 \%$ of the participants strongly agreed that social workers provide valuable assistance to cure drug addiction while $16 \%$ of the participants only agreed with the statement. However, $12.5 \%$ of the participants gave neutral response whereas an aggregate of $10 \%$ of the participants disagreed with the statement.

Table 5 Social workers comprehend needs of drug addicts for developing effective treatment strategy

\begin{tabular}{|c|c|c|}
\hline Response & Frequency & Percentage (\%) \\
\hline Strongly Agree & 105 & 52.5 \\
\hline Agree & 76 & 38 \\
\hline Neutral & 8 & 4 \\
\hline Disagree & 7 & 3.5 \\
\hline Strongly Disagree & 4 & 2 \\
\hline Total & $\mathbf{2 0 0}$ & $\mathbf{1 0 0}$ \\
\hline
\end{tabular}

In table 4, it is illustrated that participants have provided their answers with respect to their experience in attaining services from the social workers. According to above table, $52.5 \%$ of the participants strongly agreed that social workers understand needs of the drug addicts according to which effective strategies are developed. In addition, $38 \%$ of the participants agreed to the statement while only $4 \%$ of the participants gave their neutral response. Similarly, an aggregate of $5.5 \%$ of the participants disagreed with the statement.

Table 6 Social workers have prior experience and knowledge

\begin{tabular}{|c|c|c|}
\hline Response & Frequency & Percentage (\%) \\
\hline Strongly Agree & 92 & 46 \\
\hline Agree & 15 & 7.5 \\
\hline Neutral & 67 & 33.5 \\
\hline Disagree & 16 & 8 \\
\hline Strongly Disagree & 10 & 5 \\
\hline Total & $\mathbf{2 0 0}$ & $\mathbf{1 0 0}$ \\
\hline
\end{tabular}

Table 5 indicates responses of the participants regarding experience and knowledge of social workers. According to results, it is found that $46 \%$ of the participants strongly agreed that social workers are experienced and knowledgeable in terms of their role and responsibilities while only $7.5 \%$ of the participants gave an agreeable 
response. However, $33.5 \%$ of the participants provided neutral answer. $8 \%$ of the participants disagreed with the statement whereas only $5 \%$ of the participants strongly disagreed with the fact.

Table 7 Rehabilitation centers provide opportunity to social workers to help and support drug addicts in a

\begin{tabular}{|c|c|c|}
\hline \multicolumn{2}{|c|}{ professional way } & Percentage (\%) \\
\hline Response & Frequency & 85 \\
\hline Strongly Agree & 170 & 10 \\
\hline Agree & 20 & 2.5 \\
\hline Neutral & 5 & 2.5 \\
\hline Disagree & 5 & 0 \\
\hline Strongly Disagree & 0 & $\mathbf{1 0 0}$ \\
\hline Total & $\mathbf{2 0 0}$ & \\
\hline
\end{tabular}

Table 6 represents responses of the participants with respect to rehabilitation centers providing opportunities to social workers. The results indicate that $85 \%$ of the participants strongly agreed whereas $10 \%$ of the participants agreed to the statement. However, $2.5 \%$ of the participants gave their neutral response while remaining $2.5 \%$ of the participants disagreed with the statement.

Table 8 Social workers involve families as well to train their actions and behavior against drug addicts

\begin{tabular}{|c|c|c|}
\hline Response & Frequency & Percentage (\%) \\
\hline Strongly Agree & 175 & 87.5 \\
\hline Agree & 20 & 10 \\
\hline Neutral & 5 & 2.5 \\
\hline Disagree & 0 & 0 \\
\hline Strongly Disagree & 0 & 0 \\
\hline Total & $\mathbf{2 0 0}$ & $\mathbf{1 0 0}$ \\
\hline
\end{tabular}

Table 7 shows responses regarding role of social workers in involving families of drug addicts so that they could also influence the victim effectively. In this way, $87.5 \%$ of the participants strongly agreed with the statement, $10 \%$ of the participants agreed that social workers involve families as well whereas only $2.5 \%$ of the participants gave neutral replies.

Table 9 Social workers provide special care and training to drug addicts to avoid substance use

\begin{tabular}{|c|c|c|}
\hline Response & Frequency & Percentage (\%) \\
\hline Strongly Agree & 160 & 80 \\
\hline Agree & 30 & 15 \\
\hline Neutral & 2 & 1 \\
\hline Disagree & 2 & 1 \\
\hline Strongly Disagree & 1 & 0.5 \\
\hline Total & $\mathbf{2 0 0}$ & $\mathbf{1 0 0}$ \\
\hline
\end{tabular}

Table 8 illustrates results of the question that whether social workers provide special care and training to drug addicts or not. In response to the question, majority of the participants strongly agreed i.e. $80 \%$ while $15 \%$ said that they agree to the statement. Perhaps, only $1 \%$ of the participants gave neutral response and $1 \%$ of the participants also disagreed with the statement. It can be seen that only $0.5 \%$ of the participants strongly disagreed with the question.

Table 10 Social workers establish strong relation with drug addicts for building trust and loyalty

\begin{tabular}{|c|c|c|}
\hline Response & Frequency & Percentage (\%) \\
\hline Strongly Agree & 145 & 72.5 \\
\hline Agree & 15 & 7.5 \\
\hline Neutral & 30 & 15 \\
\hline Disagree & 0 & 0 \\
\hline Strongly Disagree & 10 & 5 \\
\hline Total & $\mathbf{2 0 0}$ & $\mathbf{1 0 0}$ \\
\hline
\end{tabular}


Table 9 shows results for the relationship that social workers build with drug addicts. According to the table, $72.5 \%$ of the participants strongly agreed while $7.5 \%$ of the participants only agreed with the statement. Similarly, $15 \%$ of the participants gave their neutral response however, only $5 \%$ strongly disagreed.

\subsection{Results of Hypotheses}

For understanding the role of social workers in supporting and controlling drug addicts, three different Correctional Centers based in Karachi were explored in the research process where a number of professional social workers are hired to aid and support drug addicts. Basically, the chosen centers are famous in Karachi for providing effective resources and care to the addicts with the help of social workers from various rehabilitation centers so that they could help the addicts to recover from their intense and adverse conditions which affects environment at large. There are three rehabilitation centers that help and get involve with the Correctional centers. These rehabilitation centers are; Aas recovery centre, New Horizon Care Center, and Willing Ways. All three of them work on their own for rehabilitation purpose but when it comes to helping individuals in Correctional centers they are ready to help them.

Hypothesis 1 states that the role of social workers at Correctional centers is to provide support and healthcare services to drug addicts. From the survey results, it is also informed that majority of the social workers have expertise in controlling increased psychological disabilities that are caused by excessive use of drugs consumption. It is also witnessed that the three rehabilitation centers under research domain have employed social workers who have prior knowledge and experience in treating patients who have psychological issues and thus, get involved in activities that are life threatening at large. The evidence is also taken from Slabbert (2015) that social workers have to practice clinical concepts in recovering the state of drug victims so that they could regain their health in a supervised way. Thus, it proves that hypothesis 1 is accepted while various evidence and responses have affirmed the fact that the role of social workers is related to healthcare practitioner.

Families and community itself plays an important part in changing mental state of the drug victims because they possess special bond or relationship with each other. Moriarty et al. (2015) indicates that drug addicts are often become part of such illegal and unethical activities because of the domestic and social pressure that affects their psychological stability at large. In this way, drug addicts find drug consumption more appropriate to lessen the effects of threatening environment and attain relief. Here, families are being judged particularly because of their significant role in influencing individuals to adopt such acts that are harmful for their physical, moral, and emotional health. Similarly, Raine (2018) also states that due to lack of awareness and education in the society, majority of the people become victim of such acts that causes trouble for themselves, as well as to the community on whole. Hence, drug addicts require major attention and support from their families to control their dangerous habits in order to retain their lifestyle with secure health.

Hypothesis 2 illustrates that in every Correctional Center, social workers have to play role in assisting and training families, as well as communities, in order to help drug addicts recover from critical health state (Best et al., 2016). The responses collected from survey also indicates that almost every rehabilitation center provide facility to train and develop understanding of drug addiction to families and community so that they could participate in the social work to minimize growing trend in the society. Furthermore, it is also found in the paper developed by Zastrow (2016) that with the support and increase level of knowledge about substance use and its aftereffects, drug addicts are able to fight for their recovery easily. For this purpose, it is considered highly important to educate and train families and community at large so that they could also help in treating drug addicts by utilizing actions and behavior which are in support of controlling prevailing changes in an individual. Hence, it is proved that social workers at rehabilitation centers also provide training and development sessions to community and families to motivate them for influencing drug addicts at large. In this way, following hypothesis is also accepted.

\subsection{Conclusion}

From the research, it is explored that drug addicts are the patients who require effective treatments and counseling to regain their health. In this regard, one of the important roles is found to be of social workers who are experienced professionals in terms of treating and supporting addicts. It is explored in the research that main responsibilities of social workers are based on supporting, counseling, training, effective healthcare services, engaging families and communities, and also influencing addicts with actions to improve their state of mind and physical health. The process of data collection also supports the fact that increasing trend in the Pakistan is due to societal pressure, domestic violence, seeking satisfaction and relief, and also gaining reputation in the society. However, due to such reasons, youngsters have increasingly started consuming drugs in order to achieve such goals in less time.

The findings and results of the study suggests that Correctional centers in Karachi have employed valuable and professional social workers from rehabilitation centers who are experienced in providing care, support, and consultation effectively. The respondents of the research have clearly indicated that the role of social workers is complex but important at the same time because they intend to recover mental and physical disabilities of the victim in such a way that it influences the addict to regain health in less time. It also reveals that primary role of social worker is based on following specialized framework of the rehabilitation center on the basis of which specific strategies and decisions are taken so that drug addicts could be treated effectively. Moreover, it is also 
found that social workers have to play their role in managing families and communities as well because through this way, drug addicts tend to recover even faster and with their support, drug addicts improve their routine activities at large. Hence, the role of social workers is being investigated in the context of providing awareness and training to families and communities.

The literature in the study also emphasizes on the special treatment and care that social workers provide to drug addicts with the aim that they would get to live a health life. For this purpose, many authors have regarded the responsibilities of social workers as important and influential that help drug addicts to improve their state easily. Hence, it is concluded that in order for drug addicts and their families to recover from disturbed mental conditions and increasing consumption, rehabilitation centers must be approached so that social workers would be able to treat them through their knowledge and technical experiences.

\subsection{Recommendations}

- For policymakers, it is important to include certain points in policy that are related to educating people regarding social work and their role in improving state of drug addicts

- Policies for social workers should be amended on the basis of changed culture and increasing demand in the society so that key measures could be accounted to satisfy social, economic, and legal perspectives

- Regular training and development programs must be initiated to educate social workers regarding difference in treatment and human behavior on the basis of which certain steps are taken

- Social work must acknowledge the issues caused by excessive drug consumption

- Effective supervision and management must be accounted in rehabilitation centers so that social workers could be guided according to varying needs and desirability of drug addicts

- Critical examination must be held after each session to ensure quality and standard of social work

- More research work is required to highlight key aspects which are important to be studied in order to understand and analyze important roles of social workers in controlling drug addiction.

\section{- $\quad$ Table 11 Age Group Distribution}

\begin{tabular}{|c|c|c|}
\hline Age (years) & Frequency & Percentage (\%) \\
\hline $20-25$ & 55 & 27.5 \\
\hline $26-30$ & 100 & 50 \\
\hline $31-35$ & 25 & 12.5 \\
\hline $36-40$ & 15 & 7.5 \\
\hline 41 and above & 5 & 2.5 \\
\hline Total & $\mathbf{2 0 0}$ & $\mathbf{1 0 0}$ \\
\hline
\end{tabular}

Table 12 Gender Distribution

\begin{tabular}{|c|c|c|}
\hline Gender & Frequency & Percentage (\%) \\
\hline Male & 150 & 75 \\
\hline Female & 50 & 25 \\
\hline Total & $\mathbf{2 0 0}$ & $\mathbf{1 0 0}$ \\
\hline
\end{tabular}

\section{References}

Abramovitz, Mimi. Regulating the lives of women: Social welfare policy from colonial times to the present. Routledge, 2017.

Bandiera, Frank C., Berhanu Anteneh, Thao Le, Kevin Delucchi, and Joseph Guydish. "Tobacco-related mortality among persons with mental health and substance abuse problems." PloS one 10, no. 3 (2015): e0120581.

Best, David, Melinda Beckwith, Catherine Haslam, S. Alexander Haslam, Jolanda Jetten, Emily Mawson, and Dan I. Lubman. "Overcoming alcohol and other drug addiction as a process of social identity transition: The social identity model of recovery (SIMOR)." Addiction Research \& Theory 24, no. 2 (2016): 111-123.

Brief, Social Justice. "A Social Work Perspective on Drug Policy Reform."

Brown, Kate. Vulnerability and young people: Care and social control in policy and practice. Policy Press, 2015. Carkhuff, Robert. Toward effective counseling and psychotherapy: Training and practice. Routledge, 2017.

Crossin, Rose, Andrew J. Lawrence, Zane B. Andrews, and Jhodie R. Duncan. "Altered body weight associated with substance abuse: a look beyond food intake." Addiction Research \& Theory 27, no. 2 (2019): 7684. 
Daley, Dennis C., and Marvin D. Feit. "The many roles of social workers in the prevention and treatment of alcohol and drug addiction: A major health and social problem affecting individuals, families, and society." Social work in public health28, no. 3-4 (2013): 159-164.

Galvani, S. A. "Alcohol and other drug use: The roles and capabilities of social workers." (2015).

Gao, Huan, and Jianhong Liu. "Women's Substance Abuse and Its Impacts on Children's Early Development and Deviant Behaviors." In Women and Children as Victims and Offenders: Background, Prevention, Reintegration, pp. 239-263. Springer, Cham, 2016.

Harris, John, and Vicky White. A dictionary of social work and social care. Oxford University Press, 2018.

Holt-Lunstad, Julianne, and Bert N. Uchino. "Social support and health." Health behavior: Theory, research and practice(2015): 183-204.

Jafari, Siavash, Richard Mathias, Ronald S. Joe, Souzan Baharlou, and Ashkan Nasr. "Effect of law enforcement on drug abuse: A comparison of substance use in Pakistan, Afghanistan, Iran and Turkey." Journal of Substance Use 20, no. 4 (2015): 295-300.

Jones, Nathalie Marie. "CHALLENGES OF SOCIAL WORKERS GIVING MENTAL HEALTH SERVICES TO JUVENILES WHO HAVE BEEN INCARCERATED FOR DRUG USE." (2016).

Joshi, D., and B. S. Rathore. "Women Substance Abuse a Rising Problem in India." The International Journal of Indian Psychology 4, no. 3 (2017): 9.

Khursheed, Farkhunda, and Chandra Madhudas. "Frequency of Betel nut Addiction in Pregnant Anaemic Women and its impact on fetal outcome." JOURNAL OF THE LIAQUAT UNIVERSITY OF MEDICAL AND HEALTH SCIENCES 16, no. 3 (2017): 145-148.

Lipset, Seymour. Social organization of medical work. Routledge, 2017.

Matthew, K. J., Bibhushan Regmi, and Lopsang Dinchen Lama. "Role of Family in Addictive Disorders." International Journal of Psychosocial Rehabilitation. Vol 22 (1) 6575 (2018).

McGovern, Justine, and Stephanie Sarabia. "Substance Abuse Among Older Adults: Context, Assessment, and Treatment." In New Directions in Treatment, Education, and Outreach for Mental Health and Addiction, pp. 111-124. Springer, Cham, 2018.

Moriarty, Joet al, Mary Baginsky, and Jill Manthorpe. "Literature review of roles and issues within the social work profession in England." Professional Standards Authority, London (2015).

Munir, Sadaf, Rabia Habib, Sliha Awan, Nazia Bibi, Arooj Tanveer, Sajida Batool, and Syed M. Nurulain. "Biochemical Analysis and Association of Butyrylcholinesterase SNPs rs3495 and rs1803274 with Substance Abuse Disorder." Journal of Molecular Neuroscience (2019): 1-11.

Oreskovich, Michael R., Tait Shanafelt, Lotte N. Dyrbye, Litjen Tan, Wayne Sotile, Daniel Satele, Colin P. West, Jeff Sloan, and Sonja Boone. "The prevalence of substance use disorders in American physicians." The American journal on addictions 24, no. 1 (2015): 30-38.

Raine, Pamela. Women's Perspectives on Drugs and Alcohol: The Vicious Circle: The Vicious Circle. Routledge, 2018.

Roberts, Melinda R., Iris Phillips, Thomas D. Bordelon, and Lisa Seif. "A social worker's role in drug court." SAGE Open 4, no. 2 (2014): 2158244014535413.

Scheff, Thomas J. Being Mentally Ill: A Sociological Study. Routledge, 2017.

Schnall, Eliezer. "Social support: A role for social work in the treatment and prevention of hypertension." Einstein Journal of Biology and Medicine 21, no. 2 (2016): 50-56.

Singer, Merrill, and J. Bryan Page. The social value of drug addicts: Uses of the useless. Routledge, 2016.

Slabbert, Ilze. "Reflective learning in social work education in the field of substance abuse." Social Work 51, no. 4 (2015): 549-564.

Soomro, Shamim, Misbah Bibi Qureshi, and Farzana Baloch. "Drug Addiction Scenario In Pakistan Effects And Consequences Over Youth." Grassroots 48, no. 2 (2018).

Steketee, Gail, Abigail M. Ross, and Madeline K. Wachman. "Health outcomes and costs of social work services: a systematic review." American journal of public health 107, no. S3 (2017): S256-S266.

Swensen, Isaac D. "Substance-abuse treatment and mortality." Journal of Public Economics 122 (2015): 13-30.

Wang, Zhe, Shujuan Chen, Junning Chen, Chunfeng Xu, Zhikang Chen, Wenxu Zhuang, Xu Li, Min Zhao, and Haifeng JIANG. "A Community-Based Addiction Rehabilitation Electronic System to Improve Treatment Outcomes in Drug Abusers: Protocol for a Randomized Controlled Trial." Frontiers in Psychiatry 9 (2018): 556.

Xavier, Denis, Rajeev Gupta, Deepak Kamath, Alben Sigamani, P. J. Devereaux, Nisha George, Rajnish Joshi, Janice Pogue, Prem Pais, and Salim Yusuf. "Community health worker-based intervention for adherence 
to drugs and lifestyle change after acute coronary syndrome: a multicentre, open, randomised controlled trial." The Lancet Diabetes \& Endocrinology 4, no. 3 (2016): 244-253.

Zastrow, Charles. Empowerment Series: Introduction to Social Work and Social Welfare: Empowering People. Cengage Learning, 2016. 